\title{
Neurocysticercosis presenting as hemichorea: a case report
}

\author{
Sunil Kumar, MD, PhD, Amrutha Garikapati, MD, Roopesh Yarappa, MD, \\ Sourav Chaturvedi, MD, Anusha Gupta, MD
}

\begin{abstract}
Neurocysticercosis is a parasitic infection in the brain. Its manifestation as involuntary movements is rare. We report a case of a 77-year-old man with multiple brain cysticercosis presenting with hemichorea, who improved markedly after treatment with corticosteroids and albendazole.
\end{abstract}

Key words: Chorea; Dyskinesias; Neurocysticercosis
Department of Medicine, Jawaharlal Nehru Medical College, Datta Meghe Institute of Medical Sciences, Wardha, Maharashtra, India

Correspondence to: Sunil Kumar, Department of Medicine, Jawahar Lal Nehru Medical College, Datta Meghe Institute of Medical Sciences, Wardha, Maharashtra, India. Email: sunilkumarmed@gmail.com

\section{INTRODUCTION}

Neurocysticercosis is an uncommon manifestation of a parasitic infection in the central nervous system caused by the larval form of the tape worm Taenia solium. ${ }^{1}$ Although this parasite is endemic in most part of the world, hemichorea caused by cysticercosis of the central nervous system is rare. ${ }^{2}$ Few case reports have been published on involuntary movements secondary to neurocysticercosis. ${ }^{1-3}$ We report a case of multiple neurocysticercosis of the brain in which hemichorea was the presenting feature.

\section{CASE PRESENTATION}

In March 2019, a 77-year-old man presented with a 15-day history of focal involuntary movements in his right upper limb. The patient reported no history of fever, drug use (antiemetics or antipsychotics), seizures, chronic cough, diarrhoea, weight loss, decreased appetite, or tuberculosis. He was not screened for hypertension and diabetes. On examination, the patient was conscious and lucid, but anxious. He had mild right shoulder pain secondary to the involuntary movements. His blood sugar level was $120 \mathrm{mg} / \mathrm{d}$ and blood pressure was 120/70 mm $\mathrm{Hg}$ in left upper limb supine position. The patient had haemoglobin level of $10.5 \mathrm{~g} / \mathrm{dL}$ and total leucocyte count of 8800 , with $70 \%$ polymorphs, $28 \%$ lymphocytes, and $2 \%$ eosinophils. The erythrocyte sedimentation rate was $14 \mathrm{~mm} /$ hour. The patient's renal and liver function test results were normal, and enzyme-linked immunosorbent assays for HIV 1 and 2 were both negative. His thyroid profile was normal.

Central nervous system examination results were unremarkable, except for abnormal involuntary movement of the right upper limb typical of hemichorea. The patient had choreiform movements in the right upper limb, which were primarily visible during motor tasks such as eating and writing (the patient was unable to hold a pen). Coordination was affected by hemichorea on the right side but was normal on the left side. The patient had no muscle weakness, his sensory examination results were normal, and his gait was normal.

Plain and post-contrast magnetic resonance images of the brain showed multiple cysts in the cortex, subcortex, and basal ganglia, with surrounding oedema and calcified scars suggestive of neurocysticercosis (FIG).

The diagnosis of neurocysticercosis was confirmed by western blot analysis, which revealed specific antibodies to Taenia solium, although the patient's stool was negative for parasites. The patient was treated with albendazole $400 \mathrm{mg}$ twice a day and prednisolone $1 \mathrm{mg}$ per $\mathrm{kg}$ body weight once per day. After 1 week, the patient's abnormal movements had markedly improved and he was discharged home. At the 1-month follow-up, magnetic resonance images of the brain showed marked reduction in the neurocysticercosis. 


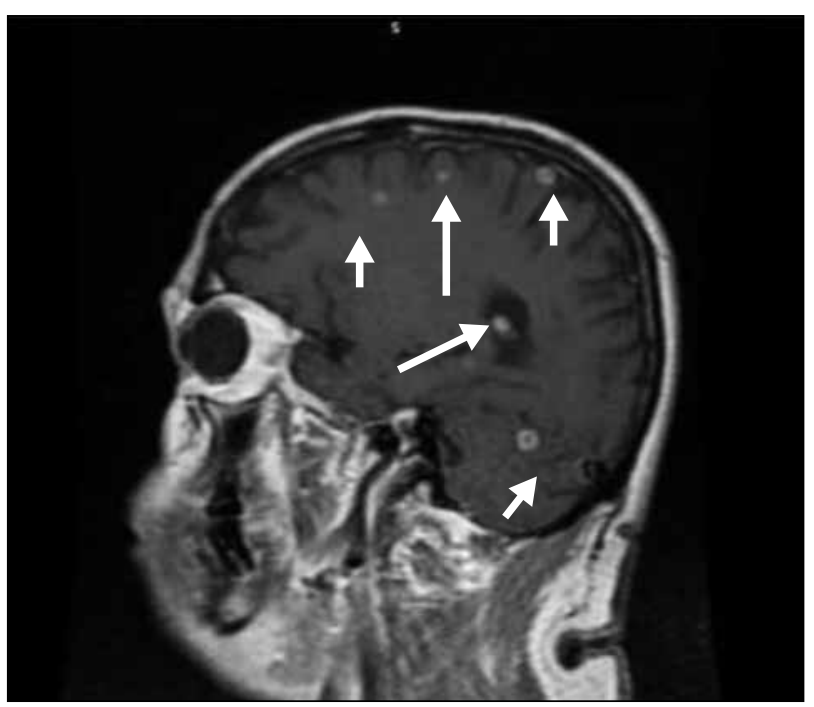

FIGURE. Magnetic resonance image of the brain showing multiple cysts with surrounding oedema (long arrow) and calcified scars suggestive of neurocysticercosis (arrows).

\section{DISCUSSION}

Hemichorea is involuntary, hyperkinetic disorder consisting of sudden irregular purposeless random movements involving one half of the body. Although the movements are purposeless, the patients may incorporate them into a deliberate act, to make them less noticeable. ${ }^{3,4}$ Hemichorea can be caused by various processes involving the nuclei of the basal ganglia, including stroke, primary and secondary malignancy, paraneoplastic syndrome, multiple sclerosis, tuberculoma, neurocysticercosis, or toxoplasmosis. ${ }^{5,6}$ The mechanisms include inflammation, hydrocephalus, mass effect, and ischaemia of the basal ganglia or related structures owing to secondary vasculitis. ${ }^{4}$

In the present case, hemichorea decreased immediately after treatment with corticosteroids and antiparasitic therapy. Thus, the responsible mechanism was the inflammatory reaction surrounding the cyst. Inflammation around a cysticercus marks the onset of the process of cyst death; therefore, some authors have recommended not using antiparasitic drugs in patients with symptomatic cysts. ${ }^{4,5}$

One pathogenic mechanism of hemichorea is interruption of GABA transmission from the striatum to the globus pallidus externa. This may increase neuronal activity in the globus pallidus externa and inhibit the subthalamic nucleus. This inhibition results in the subthalamic nucleus losing control of the globus pallidus interna neurons, leading to disinhibition of the motor thalamus. ${ }^{5}$ It remains unclear why our patient experienced only hemichorea and no other neurological symptoms. Further studies using functional or perfusion images are warranted to elucidate the pathophysiology of hemichorea, especially in neurocysticercosis involving uncommon sites in the brain.

\section{DECLARATION}

The authors have no conflict of interest to disclose.

\section{REFERENCES}

1. Cosentino C, Vélez M, Torres L, Garcia HH. Neurocysticercosisinduced hemichorea. Mov Disord 2006;21:286-7. Crossref

2. Verma R, Agarwal A, Kar AM. Hemichorea resulting from single enhancing computed tomography lesion. J Assoc Physicians India 2006;54:735-7.

3. Bhatia R, Desai S, Garg A, Padma MV, Prasad K, Tripathi M. Isolated facial myokymia as a presenting feature of pontine neurocysticercosis. Mov Disord 2008;23:135-7. Crossref

4. Chung SJ, Im JH, Lee MC, Kim JS. Hemichorea after stroke: clinical-radiological correlation. J Neurol 2004:251:725-9. Crossref

5. Kumar S, Joshi R, Jain AP. Hemichorea: a rare presentation of acute stroke. J Int Med Sci Acad 2008;21:81.

6. Kumar S, Jain S, Kashikar S. Herculean appearance due to disseminated cysticercosis: case report. Asian Pac J Trop Med 2012;5:1007-8. Crossref 